This item was submitted to Loughborough's Research Repository by the author.

Items in Figshare are protected by copyright, with all rights reserved, unless otherwise indicated.

\title{
Biomimetic locomotion on water of a porous natural polymeric composite
}

PLEASE CITE THE PUBLISHED VERSION

http://dx.doi.org/10.1002/admi.201500854

PUBLISHER

(c) Wiley

VERSION

SMUR (Submitted Manuscript Under Review)

LICENCE

CC BY-NC-ND 4.0

REPOSITORY RECORD

Liakos, loannis, Pietro Salvagnini, Alice Scarpellini, Riccardo Carzino, Elisa Mele, Vittorio Murino, and Athanassia Athanassiou. 2016. "Biomimetic Locomotion on Water of a Porous Natural Polymeric Composite". figshare. https://hdl.handle.net/2134/20820. 


\section{Advanced Materials Interfaces \\ Bio-mimetic locomotion on water of a porous natural polymeric composite \\ --Manuscript Draft--}

Manuscript Number:

Full Title:

Article Type:

Keywords:

Corresponding Author:

\begin{tabular}{|l}
\hline Additional Information: \\
\hline Question \\
\hline $\begin{array}{l}\text { Corresponding Author Secondary } \\
\text { Information: }\end{array}$ \\
\hline Corresponding Author's Institution: \\
\hline Corresponding Author's Secondary \\
\hline Institution:
\end{tabular}

First Author:

First Author Secondary Information:

Order of Authors:

(n)

Order of Authors Secondary Information:

Abstract: admi.201500373

Bio-mimetic locomotion on water of a porous natural polymeric composite

Full Paper

Self-Motors, Bio-locomotion, Bio-mimetics, Marangoni effect, Surface tension gradient.

loannis Liakos

Istituto Italiano di Tecnologia

Genova, ITALY

\section{Response}

Istituto Italiano di Tecnologia

loannis Liakos

loannis Liakos

Pietro Salvagnini

Elisa Mele

Riccardo Carzino

Alice Scarpellini

Athanassia Athanassiou

Observation of the natural world can provide invaluable information on the mechanisms that semi-aquatic living organisms or bacteria use for their selfpropulsion. Microvelia, for example, uses wax excreted from its legs to move on water in order to escape from predators or reach the bank of the river. Mimicking such mechanism, few self-propelled materials on water, as camphor, have been previously developed, but weak points like slow locomotion, short movement duration or shaperestrictions still need to be improved. Herein we present a totally green self-assembled porous system, formed by the combination of a natural polymer with an essential oil, that spontaneously moves on water for hours upon expulsion of the oil, with high velocities reaching $15 \mathrm{~cm} / \mathrm{s}$. The structural characteristics of the natural polymeric composite are carefully analyzed and associated to its spontaneous movement. Surface tension change experiments are also presented that connect the essential oil release with the locomotion of the porous composite films. This research work opens novel routes towards bio-inspired natural materials that can be used for mimicking and studying the motion of bio-organisms and microorganisms, and for applications such as energy harvesting, aquatic pollution monitoring, drug delivery, to name few. 
DOI: $10.1002 /$

Article type: Full paper

\section{Bio-mimetic locomotion on water of a porous natural polymeric composite}

Ioannis Liakos*, Pietro Salvagnini, Elisa Mele, Riccardo Carzino, Alice Scarpellini, Athanassia Athanassiou*

Dr. I. Liakos, Dr. E. Mele, Dr. R. Carzino, Dr. A. Athanassiou

Smart Materials, Istituto Italiano di Tecnologia (IIT), via Morego 30, 16163 Genova, Italy.

Emails: ioannis.liakos@iit.it, athanassia.athanassiou@iit.it

Dr. Pietro Salvagnini

Pattern Analysis and Computer Vision, Istituto Italiano di Tecnologia (IIT), via Morego 30, 16163 Genova, Italy.

Dr. A. Scarpellini

Nanochemistry, Istituto Italiano di Tecnologia (IIT), via Morego 30, 16163 Genova, Italy.

Keywords: Self-Motors, Bio-locomotion, Bio-mimetics, Marangoni effect, Surface tension gradient.

Observation of the natural world can provide invaluable information on the mechanisms that semiaquatic living organisms or bacteria use for their self-propulsion. Microvelia, for example, uses wax excreted from its legs to move on water in order to escape from predators or reach the bank of the river. Mimicking such mechanism, few self-propelled materials on water, as camphor, have been previously developed, but weak points like slow locomotion, short movement duration or shape-restrictions still need to be improved. Herein we present a totally green self-assembled porous system, formed by the combination of a natural polymer with an essential oil, that spontaneously moves on water for hours upon expulsion of the oil, with high velocities reaching $15 \mathrm{~cm} / \mathrm{s}$. The structural characteristics of the 


\section{Introduction}

Motion of biological systems can be induced from non-equilibrium conditions, related to physical or chemical instabilities. ${ }^{[1,2]}$ When the driving force is the surface tension gradient created at the edges of floating systems in contact with water, then the movement is explained by the so-called "Marangoni effect". ${ }^{[3-6]}$ Non-biological self-propelled systems based on this effect have also been developed, with the most studied one being the camphor that shows spontaneous movement on the air/water interface. ${ }^{[1,}$ ${ }^{7-25]}$ Other self-motors with propelling function based on the same principle have been reported and can

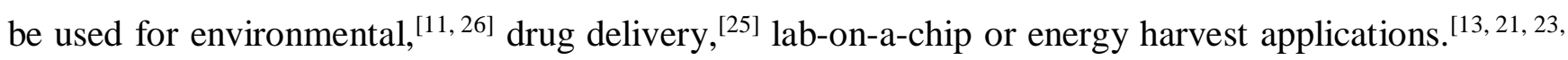
24] The maximum recorded velocity of a camphor disk on the air/water interface has been approximately $6 \mathrm{~cm} / \mathrm{s}^{[10]}$ with the duration of the motion lasting few seconds. Disk-shaped gel particles formed from camphor, agarose and PDMS were also developed for spontaneous locomotion at the air/water interface ${ }^{[22]}$ with maximum velocity $2 \mathrm{~cm} / \mathrm{s}$. A different than camphor system that has been reported was based on a 1,10-phenanthroline disk moving on divalent metal ion aqueous solutions, where the velocity of the disk ranged from 10 to less than $3 \mathrm{~cm} / \mathrm{s}$ depending on the metal ions. ${ }^{[15]}$ Another reported system was made of self-assembled peptides incorporated into a metal-organic framework and released upon contact with water, causing a propulsion with velocity between 4.5 and $8.5 \mathrm{~cm} / \mathrm{s}$ for few minutes. ${ }^{[27]}$ In an alternative approach the supply of ethanol to a tank reservoir on the 
top of a nanocellulose film provided surface tension gradient on the water surface, responsible for the movement of the film for $74 \mathrm{~m}$ at a velocity of nearly $2 \mathrm{~cm} / \mathrm{s}\left(\sim 1\right.$ hour movement) ${ }^{[9]}$ Additionally, lithographically structured poly-N-isopropylacrlylamide (PNIPAm) gels soaked in ethanol and then placed on water have shown velocities of $17-39 \mathrm{~cm} / \mathrm{s}$ depending on the shape and size of the gel. ${ }^{[7]}$ Similar velocities $(30 \mathrm{~cm} / \mathrm{s})$ have been also reached with microflotillas consisting of various SU-8 microboats filled with isopropyl alcohol and linked with a Nylon rope ${ }^{[12]}$ An overall review of the various self-propelled systems (either those where the propellant container was at the same time the propelled object or the ones where the propellant was placed in a reservoir) and their velocities is well described in the work of Pimienta and Antoine. ${ }^{[28]}$

Here, we present the realization of a new self-propelled system consisting of cellulose acetate (CA) and peppermint (PM) essential oil. Disc-shaped objects of few millimeters diameters made of this natural composite are capable of producing significant mechanical work on the air/water interface for few hours. In particular, upon contact with water the presented natural composites start expelling the contained essential oil changing their composition in a dynamic way. The movement of the system is due to imbalance of forces parallel to the water surface, because of differences of surface tension created at the margins of the samples due to the essential oil expel. Apart from the PM essential oil release in water, also its simultaneous partial evaporation in air is responsible for the surface tension gradients created at the edges of the floating object at the air/water interface. The Marangoni flow that pulls the composites from their position occurs from the lower (expelled essential oil) to the higher (water) surface tension areas. ${ }^{[29]}$ The composite developed in this work has many advantages compared to other systems since it is totally natural and inexpensive, self-assembles into a porous structure, can be used as self-standing material or as coating on devices, its direction of motion can be selected by modifying its shape, its propulsion can have very long duration and reaches velocities higher than 10 $\mathrm{cm} / \mathrm{s}$. 


\section{Methods and Results}

\subsection{Composite Material Development}

Free-standing composite films of CA and PM essential oil were developed as described in the experimental part and subsequently were accurately cut in disc shapes of $14 \mathrm{~mm}$ diameter, $55 \mu \mathrm{m}$ thickness and $10 \mathrm{mg}$ weight. The calculated amount of PM oil inside the Motor was $6.7 \mu \mathrm{g}$. The CAPM essential oil disc Motors attain a whitish color, different than the transparent bare CA film (Figure 1a), due to light scattering effects caused by their structure, that will be analyzed further down. The Motors are flexible (Figure 1b) and are floating on water (Figure 1c). Investigations by atomic force microscope $(\mathrm{AFM})$ revealed the presence of distinct spherical domains of approximately $10 \mu \mathrm{m}$ in diameter exposed on the surface of the polymer matrix (Figure 1d,e). This is also confirmed by scanning electron spectroscopy (SEM) images, where the porosity of the samples is clearly visible not only on the surface (Figure 1f) of the films, but also throughout their volume (Figure 1g). This porosity is responsible for the whitish color of the composite samples due to light scattering. The reason of the formation of this porous structure is the spontaneous phase separation between the PM essential oil and the CA polymer, causing the creation of PM microspheres throughout the polymer volume. During the drying of the films, acetone, the volatile co-solvent of the two components of the samples evaporates, and the oil micro-droplets remain as filled pores in the matrix. Therefore, all the pores revealed during the AFM and SEM investigation are filled with the PM essential oil. 
Figure 1 Demonstration of the Motors and their topography. a, Comparison between bare CA (left) and CA-PM Motor (right) film. b, Demonstration of the flexibility of the disc Motor. c, Photograph showing the Motor floating on water. d, Topography AFM image of the surface of a Motor. e, Phase AFM image of the surface of a Motor. f, Surface SEM image of a Motor and a zoom in a pore for better visualisation (inset). g, Cross-section SEM image of a Motor and a higher magnification zoom (inset).

\subsection{Self-propulsion on Water}

Upon placement of the microporous disc-shaped Motors on the water surface, they exhibit intense and variable propulsion. The locomotion of the disc Motors, has been recorded using a video camera and analyzed using recognition and edge detector techniques. ${ }^{[30-33]}$ The analyzed motion of one Motor shown in Figure 2, demonstrates different motion patterns on the water, varying between asymmetric (both axes of motion were irregular) (Figs 2a, 2c and 2d) and symmetric (one of the two axes was constant) (Figure 2b) and between continuous (Figure 2a) and intermittent (Figure 2d). In general, arbitrary motion of the Motors can be attributed to increased excretion of the essential oil from 


\section{WILEY-VCH}

selected sites of the Motor with respect to others that changes dynamically, due to structural variations in the films, creating varying surface tension barriers at the Motors' edges that need to be overcome from the Motors in order to move, all phenomena that will be further analyzed next. The position of the Motor versus time is plotted in Figure 2e for the whole motion duration, where it can be seen that for most of the time the motion was quite regular and was happening on average around all the area of the $14 \mathrm{~cm}$-diameter Petri dish. Only in the beginning (0-320 s) and towards the end, after approximately $8200 \mathrm{~s}$, the motion appeared more random and localized at one side of the Petri dish. In the latter case the motion becomes localized due to reduced mobility of the Motor, whereas in the former case the reason can be connected to the highly irregular essential oil release at early times. In Figure $2 \mathrm{f}$ is shown the position versus time of the Motor with respect to the center of the Petri dish for these early locomotion times (first 320 s), where are highlighted with yellow the time intervals illustrated in Figure $2 \mathrm{a}, 2 \mathrm{~b}$ and $2 \mathrm{c}$. The relative graphs of position versus time for all Figures $2 \mathrm{a}-2 \mathrm{~d}$ are shown in the supporting information (Figure S1).

The velocity of the Motor in both $\mathrm{x}$ and $\mathrm{y}$ directions reaches its maximum values, close to 15 $\mathrm{cm} / \mathrm{s}$, in the time period between about $700 \mathrm{~s}(12.5 \mathrm{~min})$ and $1500 \mathrm{~s}(25.0 \mathrm{~min})$ of the propulsion (Figure 2g). This velocity is higher than those of other reported Motors where, as in our case, the material is the propelled object itself. For example, the velocity of a representative camphor on water was about $4 \mathrm{~cm} / \mathrm{s}^{[28,34]}$ and of a metal organic framework that was releasing peptides onto the water-air interface from 2 to $8 \mathrm{~cm} / \mathrm{s}$ for few minutes ${ }^{[27]}$. Interestingly, the disc was rotating both clockwise and anticlockwise (supporting information Figure S2), another indication of uneven distribution of the expelled essential oil along the disc periphery, with the average module of rotation velocity oscillating between 10 - $20 \mathrm{rpm}$ approximately (supporting information Figure S3). From the translational velocity (Figure $2 \mathrm{~g}$ ), the angular velocity (Figure S3) and knowing the mass $(0.01 \mathrm{~g})$ and the inertia of the disc Motor it was possible to estimate its kinetic energy, that is plotted in Figure $2 \mathrm{~h}$. It can be 
noticed that the maximum kinetic energy was not obtained at the very beginning but after almost 13 minutes $(778 \mathrm{~s})$ from the initiation of the motion. This time is most likely related to the maximization of the essential oil release rate from the CA matrix. Indeed after about 13 min of locomotion the number of discrete movements of the Motor is maximum, and subsequently it decreases with time gradually, as shown in Figure 2i. The cumulated overall distance travelled by the Motor was calculated integrating the velocity of the Motor (Figure $2 \mathrm{~g}$ ) and is shown in Figure $2 \mathrm{j}$. After $3 \mathrm{~h}$ the disc, that contained $6.7 \mathrm{mg}$ of PM, had travelled more than $77 \mathrm{~m}$, a much higher distance than the one travelled by camphor boats (millimetres or centimetres), ${ }^{[1,28,34]}$ by polymeric capsules with organic solvents $(25$ $\mathrm{m})^{[35]}$ and by flotilla boats with isopropyl alcohol reservoir $(1 \mathrm{~m})^{[12]}$. In the work of metal organic framework the amount of peptides used as a fuel was varying between 234 and $702 \mathrm{mg}$ and the framework was propelled with velocities ranging from 2 to $8 \mathrm{~cm} / \mathrm{s}$ for $5-15 \mathrm{~min}$ depending on the amount of peptides ${ }^{[27]}$. In another work, $5 \mu \mathrm{l}$ of a polysulfone in N,N-dimethylformamide (DMF) solution was placed on a water surface and solidified creating porous polymer capsules. The DMF was slowly released ranging the velocity between $11.4-15.7 \mathrm{~cm} / \mathrm{s}$ and for a distance of $24.52 \mathrm{~m}$. 

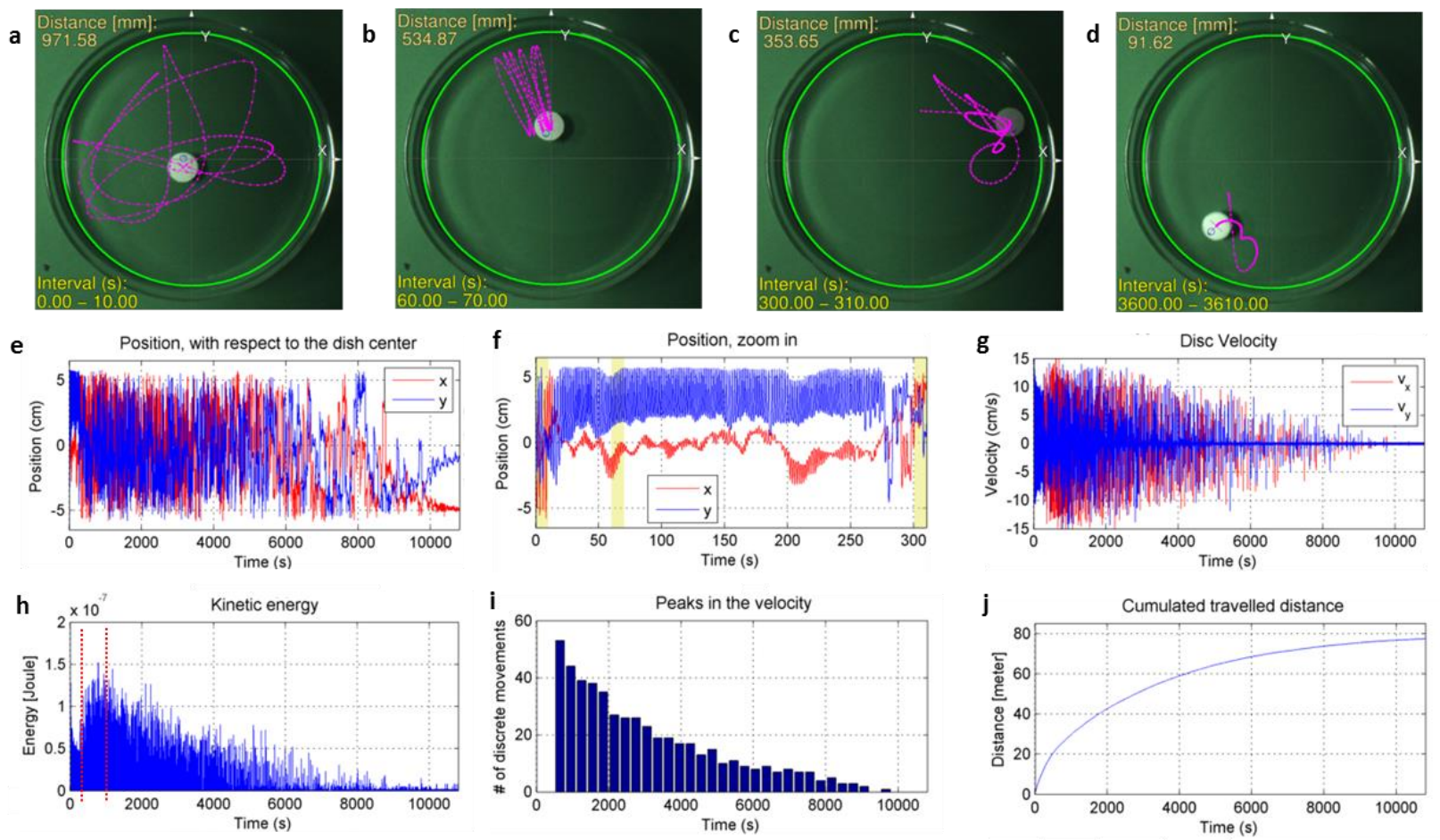

Figure 2 Motion analysis of disc Motor. a-d, Locomotion of Motor and trajectory of its motion (pink line) at different time intervals of $10 \mathrm{~s}$ from the beginning of the experiment until $1 \mathrm{~h}$ and $10 \mathrm{~s}$. e, Position versus time of the Motor with respect to the centre of the Petri dish and along the $\mathrm{x}$ and $\mathrm{y}$ directions. f, Zoom of the position of the Motor versus time for the first $320 \mathrm{~s}$, where the highlighted yellow zones correspond to the trajectory motions shown in a, b and c. g, Instantaneous (computed between 3 consecutive frames) velocity of the disc motor from the centre of the Petri dish and along the two axes during time. $\mathbf{h}$, Kinetic energy of the Motor, both from disc translation and disc self-rotation. i, Number of distinct motions of 5 min intervals. $\mathbf{j}$, Cumulated distance travelled by the Motor versus time.

The motion termination of the CA-PM Motor can be related to both the total expulsion of the essential oil from the porous composite and the saturation of the surface tension. The second hypothesis 


\subsection{Mechanism of Locomotion}

was verified in this work since for all the experiments performed onto the confined water surface, after the end of the propulsion effect, the samples were able to continue the movement when they were put onto fresh water. This demonstrates that even if a part of the released essential oils evaporates, the substances remaining onto but also dissolved into the water would eventually minimize the surface tension differences between the expelled oil and the liquid on which the Motor floats, preventing the locomotion of the latter. Two videos (see supporting materials Video S1) show the aged disc-shaped Motor after $2 \mathrm{~h}$ and after 1 day locomotion on water, respectively, dried and placed onto fresh water (note that disc Motors might need to be flatten on the water prior to their movement, since they were slightly bended when dried). The videos of selected intervals of the Motor are presented in the supporting information (Videos S2-S8). During the first phase of the experiment (before removal from the water and drying) the motion of the Motor after 2 hours was very slow with velocity from $0-60$ $\mathrm{mm} / \mathrm{s}$ and just 3 intermittent movements within 2 min (Video S8), whereas after one day the sample appeared immobile. Inversely, the Motor previously left for $2 \mathrm{~h}$ on water, after being dried and repositioned on fresh water, was able to move again in a continuous way (Video S1 - left part of the video) for the first minutes and intermittently afterwards, completing its motion after $1600 \mathrm{~s}(27 \mathrm{~min})$ approximately. Noticeably the motion was always regular following the path of boundaries of the Petri dish, most probably due to the low amount of remaining PM in the CA matrix that was continuously released towards one side indicated by the motion direction. The aged Motor previously left for 1 day on water was able to perform just few small movements on the fresh water (Video S1 - right part of the video) since most of its pores, previously containing the essential oil, were already open, as is described below in the SEM analysis section. 
In Figure 3 the SEM topography of the Motors before and after the propulsion are illustrated. The number of open surface pores considerably increased after 1 day of Motor floating on the water (Figure $3 a$ and $3 b$ ). Figure $3 c$ shows a slightly-open pore of an as-prepared Motor, whereas Figure $3 d$ shows an open pore after 1 day of the Motor on water, indicating how a closed pore can become open after the excretion of the essential oil onto the water. It was noted that some of the pores were already open before deposition of the Motor on the water surface (Figure 3a and 3e). The release of the oil after the contact of the composite films with the water can be attributed to the slightly hygroscopic character and therefore swelling of the matrix of the Motor that might cause the opening of the pores releasing the entrapped essential oil (schematically illustrated in Figure 3f, showing also the expected volatility of PM oil). As shown before, both the velocity and the kinetic energy of the Motors need few minutes to arrive to their maximum, and during these initial minutes the motion of the Motors is more confined with respect to consequent times (Figure 2e). These minutes most likely coincide with the time needed for the optimization of the swelling of the matrix, and thus the maximization of the release rate of essential oil out of it. Water contact angle measurements (WCA) on the Motors' surface before and after the end of their locomotion revealed that they have a very hydrophilic character in the first case while they become quite hydrophobic in the second (supporting information Figure S4). This increase in hydrophobicity is most likely due to opening of the pores of the film during the locomotion releasing the essential oil. At the end of the locomotion and after drying of the Motors at ambient atmosphere the air trapped inside the open pores can increase dramatically their surface hydrophobicity. ${ }^{[36]}$ 

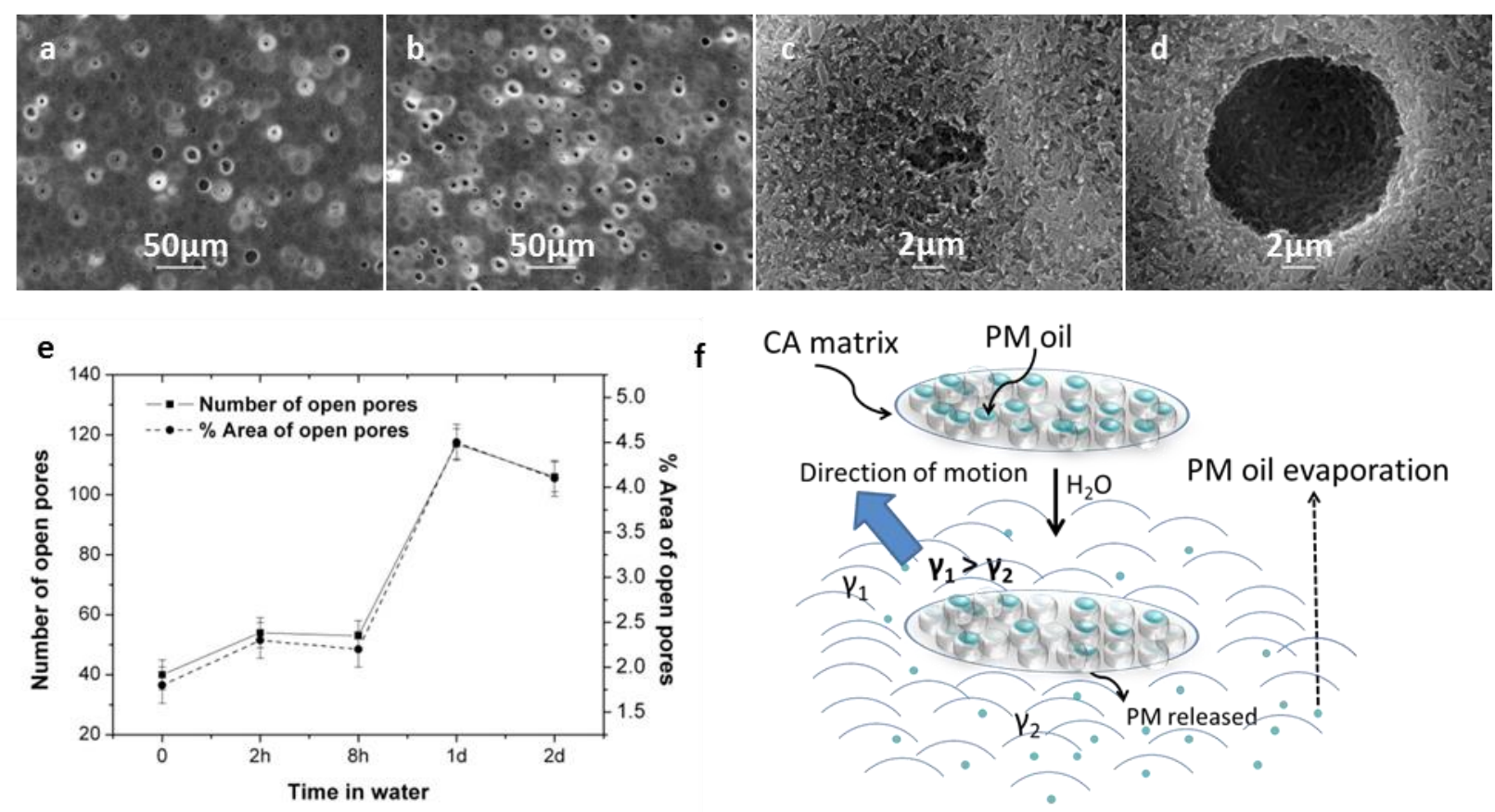

Figure 3 Release of PM oil from the Motor on water and mechanism of locomotion. a, SEM of the surface of an as-prepared Motor. b, SEM of the surface of the same Motor after 1 day on water. c, SEM of a slightly open pore at the surface of an as-prepared Motor. d, SEM of an open pore at the surface of the Motor after 1 day on water. e, Number of pores and their \% area with respect to the area of the matrix calculated using ImageJ analysis from SEM images taken at different times of the Motor on the air/water interface. $\mathbf{f}$, Schematic illustration of PM oil release/evaporation and surface tension gradient mechanism $\left(\gamma_{1}>\gamma_{2}\right)$ that describes the phenomenon of the Motor motion.

To explain in detail the locomotion mechanism, changes in the water surface tension were measured using a Langmuir-Blodgett (LB) tensiometer after placing the Motor on the surface of $200 \mathrm{ml}$ of water in a Petri dish of $14 \mathrm{~cm}$ diameter. It is expected that as soon as the Motor comes in contact with the water the PM essential oil starts being released decreasing the surface tension of water, since PM has much lower surface tension (approximately $27 \mathrm{mN} / \mathrm{m}$ ) than water $(72 \mathrm{mN} / \mathrm{m}) .{ }^{[37]}$ The changes in the surface tension do not depend only on the essential oil release but on a combination of subsequent 

and few water soluble chemical compounds. ${ }^{[38]}$

The change in the surface tension $(\pi(\mathrm{t}))$ that in the bibliography is often found as surface pressure $^{[39,40]}$ is given by the Equation 1:

$$
\pi(t)=\left|\gamma_{0}-\gamma(t)\right|
$$

where $\gamma_{0}$ is the surface tension of pure water and $\gamma(\mathrm{t})$ is the surface tension related to the essential oil's release, evaporation and dissolution. After the placement of the Motor on the water surface, the change of surface tension increased rapidly reaching about $2 \mathrm{mN} / \mathrm{m}$ in $700 \mathrm{~s}$ (Figure $4 \mathbf{a}$ and Figure S5 in supporting information). After the first $700 \mathrm{~s}$, the variation in the surface tension with respect to the initial water surface tension started decreasing slowly showing sharp peaks with decreasing frequency in time (Figure 4c) until approximately 8000 s. After that, the peaks' occurrence stopped almost completely, and the change in surface tension reached eventually the value $1.25 \mathrm{mN} / \mathrm{m}$ approximately after $3 \mathrm{~h}$. The monitored peaks are most likely related to water-induced disturbances to the probe of the tensiometer due to the discrete movements of the Motor, indicating change in the direction of the motion, triggered by essential oil accumulation at a specific sample side. Indeed, in Figure $2 \mathrm{i}$ presented above it is clearly demonstrated that the number of discrete motions of the Motor is minimized after 8000 s. Moreover, comparing the behavior of the surface tension change vs. time (Figure 4a) with that of the Motor's kinetic energy vs time presented above (Figure 2h) it can be seen that the maximum values in both cases are reached approximately $700 \mathrm{~s}$ after the initiation of the Motor's motion, verifying that the motion of the Motor depends on the changes of the surface tension at its borders induced by essential oil release. As expected, the control experiment with the CA disc without the PM essential oil placed on water, showed that the variation of surface tension remained zero with just a small peak due to the disturbance of the water surface upon placement (Figure 4a blue line). 
The combinatory effect of the sequential or parallel events of the essential oil-release from the CA matrix, its partial evaporation, and dissolution, is responsible for the dynamic change of the surface tension of the liquid on which the Motor is moving. Even after $3 \mathrm{~h}$ of motion (Figure $4 \mathrm{a}$ ) the surface tension has not reached a stable value. This dynamic change is responsible for the prolonged movement of the CA-PM Motors, since as long as the surface pressure is not stabilized the propulsion continues. Indeed, in the case of camphor boats ${ }^{[41,42]}$ or soaps ${ }^{[43]}$ the surface pressure reaches rapidly a plateau resulting in their short motion duration (seconds or minutes). Comparing the camphor and the soap systems, the use of the first saturates faster the surface with surfactants than the more volatile camphor, making the motion of the second to last longer ${ }^{[44]}$.

Figure $4 \mathrm{~b}$, that shows in detail the changes in surface tension for the first $300 \mathrm{~s}$ of motion of the composite Motor, reveals that during this time this change increases in a continuous, undisturbed way. Figs. $4 \mathrm{c}, \mathrm{d}$ instead show in detail the changes in surface tension for later times of the motion and demonstrate that the surface tension variation shows repeated "spikes" with decreasing frequency as the time passes. As mentioned above, the "spikes", that start to occur after $300 \mathrm{~s}$ of motion, represent discrete movements of the natural composite, since they result from the perturbation on the water surface every time the Motor changes direction. The "spikes" reveal a motion of the Motor with increased intensity after $300 \mathrm{~s}$, exactly as described above in the Figure 2. This most likely occurs because the CA matrix needs this initial time to swell by water, releasing after the swelling in a more abrupt way the encapsulated essential oil from the pores. The number of the discrete movements vs locomotion time was calculated and depicted in Figure 4e, and is found to be very similar to the one obtained from the video analysis of the experiment performed under the same conditions, previously presented in Figure 2i. 

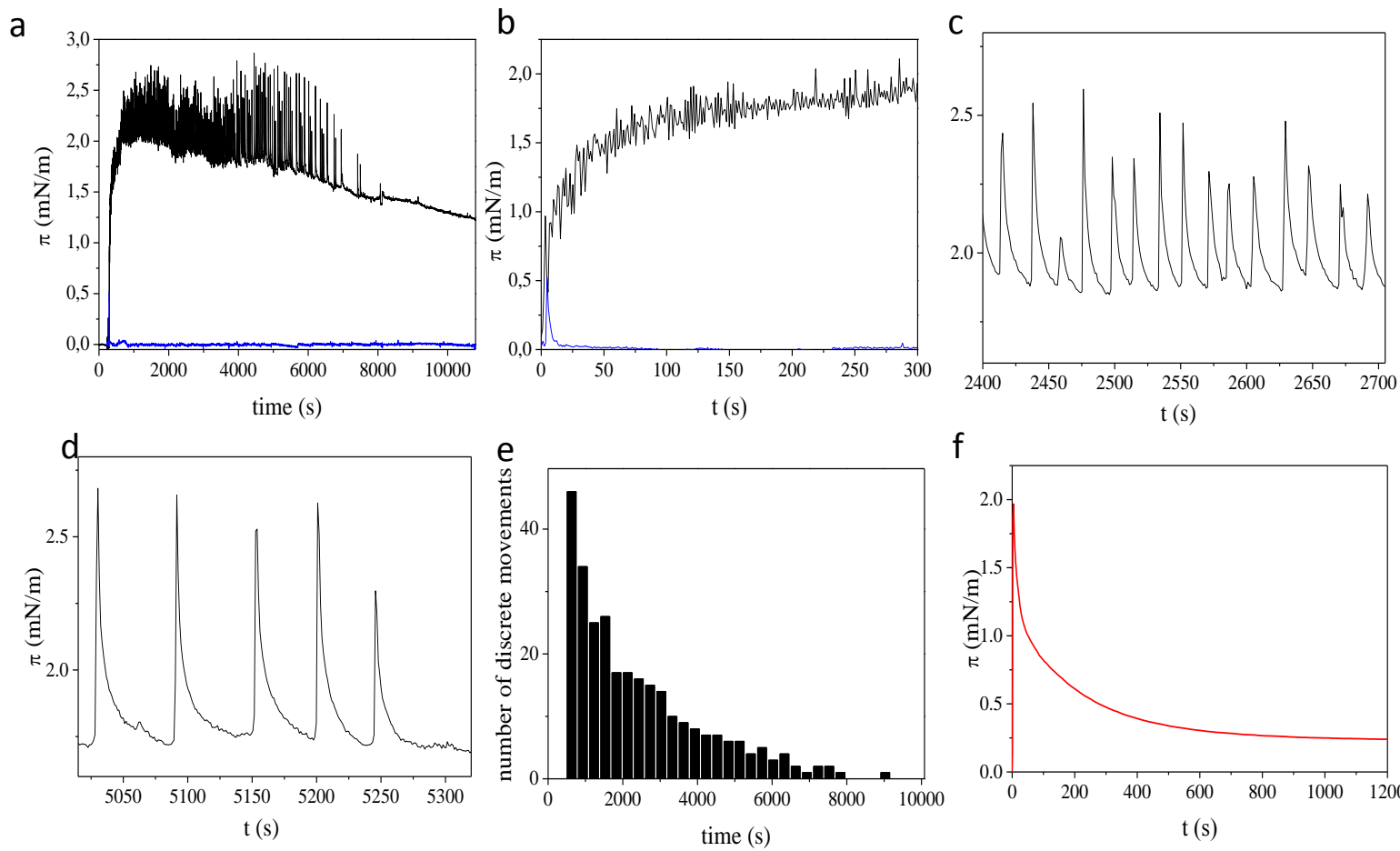

f

Figure 4 Experiments of surface tension change connected with the locomotion of the composite films and intermittent motion analysis. a, Surface tension change vs. time measurements of CA only (blue line) and CA-PM (black line) Motor at air/water interface. b, Surface tension change vs. time measurements in the continuum motion region (black line). c,d Surface tension change vs. time measurements in the intermittent motion region. e, Number of discrete movements of the Motor at 5 min time intervals. f, Relaxation experiment of $0.6 \mathrm{mg}$ of EO spread at air water interface.

In order to calculate the amount of the essential oil needed for a change in the water surface tension by $2 \mathrm{mN} / \mathrm{m}$, different amounts of PM essential oil were spread at the free air water interface (without the Motor). From the obtained surface pressure vs area isotherms depicted analytically in supporting information Figure S6 the calculated amount of PM essential oil was about $0.6 \mathrm{mg}$. In Figure $4 \mathrm{f}$ is shown the variation of the surface tension with the time, when $0.6 \mathrm{mg}$ of PM essential oil was released onto the water surface at time zero. In this case the maximum change of surface tension of 


\section{Conclusions}

Summarizing, in this work it was presented the development of a new natural porous composite material, using a combination of cellulose acetate polymer with an essential oil, that showed selfmotion on water due to the "Marangoni effect". The velocity duration of the natural composite are higher compared to other propellants, that contain the propelled object themselves, reported until now. The presented system is a versatile self-motor without the need of an external fuel reservoir or an added boat to stimulate or support the composite. The mechanism of the locomotion was based on the slow release of essential oil due to the highly porous matrix of the Motors, and its simultaneous evaporation from the water surface, resulting in extended surface tension gradient, enabling the prolonged movement of the Motor. The Motors stop moving when all the PM oil is released from their pores. An amount of $6.7 \mu \mathrm{l}$ of PM essential oil was able to move the Motors for $60 \mathrm{~m}$ in $4000 \mathrm{~s}$ (1 hour and 7 min) and for more than $75 \mathrm{~m}$ in 3 hours. A velocity of more than $10 \mathrm{~cm} / \mathrm{s}$ was achieved at least for the first 30 min of movement. It was also found that the surface tension change was very well correlated to the kinetic energy variations of the Motors in time. The knowledge acquired in this work can be useful 


\section{Experimental Section}

Incorporation of PM oil into CA and preparation of boats. A solution of CA (5\% w/v) and PM oil $(10 \% \mathrm{v} / \mathrm{v})$ in acetone was prepared. The mixture was casted on a silicon wafer and the solvent was left to evaporate. Then the film was detached from the substrate and cut in disc shapes with $14 \mathrm{~mm}$ diameter, $55 \mu \mathrm{m}$ thickness and $10 \mathrm{mg}$ weight.

Instrumentation. A Park System AFM instrument (XE-100) was used in non-contact mode. The images were acquired in air on an anti-vibration table (Table Stable TS-150) and within an acoustic enclosure. Single-beam silicon cantilevers tips (PPP-NCHR-10) were used for the data acquisition with about less than $10 \mathrm{~nm}$ nominal radius and $42 \mathrm{~N} / \mathrm{m}$ elastic force constant for high sensitivity. The resonance frequency was defined around $280 \mathrm{kHz}$ and the scan rate was maintained at $0.2 \mathrm{~Hz}$. SEM was carried out using a JEOL JSM6490LA (Jeol, Tokyo, Japan) equipped with a W thermionic source working at an acceleration voltage of $15 \mathrm{kV}$. Imaging has been obtained using secondary electrons. Due to the nonconductive nature of the samples, they have been coated with a $10 \mathrm{~nm}$ gold layer using a Cressington 108 Auto sputter coater (Cressington Scientific Instruments Inc, Cranberry Twp, PA). Static surface isotherm and surface -area isotherms experiment were performed with a KSV 2000 Langmuir equipped with a platinum balance with $4 \mu \mathrm{N} / \mathrm{m}$ of resolution. For the static surface measurements, the Motors were placed in contact with the surface of $200 \mathrm{ml}$ of Milli-Q water $(\mathrm{pH}=7.55)$ contained in a circular glass Petri dish with an internal diameter of $14 \mathrm{~cm}$. The surface -area isotherms were performed on a PTFE trough with $280 \mathrm{ml}$ of Milli-Q water as subphase. Wettability measurements were carried out by sessile drop method using a DataPhysics OCAH 200 Contact Angle 


\section{Supporting Information}

Supporting Information is available from the Wiley Online Library or from the authors.

\section{Acknowledgements}

The Authors would like to thank Dr. Ilker S. Bayer for his useful discussions at the initial stages of this work and Prof. Vittorio Murino for his helpful discussions on the video analysis of the motion

Received:

Revised: 


\section{References}

1. Nakata, S.,Y. Hayashima, Journal of the Chemical Society-Faraday Transactions 1998, 94, 3655.

2. Sempels, W., R. De Dier, H. Mizuno, J. Hofkens, J. Vermant, Nat Commun 2013, 4, 1757.

3. Gao, X.,L. Jiang, Nature 2004, 432, 36.

4. $\quad$ Bush, J.W.M., D.L. Hu, M. Prakash, Advances in Insect Physiology 2007, 34, 117.

5. Dickinson, M., Nature 2003, 424, 621.

6. Hu, D.L., B. Chan, J.W. Bush, Nature 2003, 424, 663.

7. Bassik, N., B.T. Abebe, D.H. Gracias, Langmuir 2008, 24, 12158.

8. Ikura, Y.S., R. Tenno, H. Kitahata, N.J. Suematsu, S. Nakata, J Phys Chem B 2012, 116, 992.

9. Jin, H., A. Marmur, O. Ikkala, R.H.A. Ras, Chemical Science 2012, 3, 2526.

10. Kitahata, H., S. Hiromatsu, Y. Doi, S. Nakata, M.R. Islam, Physical Chemistry Chemical Physics 2004, 6, 2409.

11. Liu, X., J. Gao, Z. Xue, L. Chen, L. Lin, L. Jiang, S. Wang, ACS Nano 2012, 6, 5614.

12. Luo, C., H. Li, L. Qiao, X.C. Liu, Microsystem Technologies-Micro-and Nanosystems-Information Storage and Processing Systems 2012, 18, 1525.

13. Mou, F., C. Chen, H. Ma, Y. Yin, Q. Wu, J. Guan, Angew Chem Int Ed Engl 2013, 52, 7208.

14. Nagayama, M., S. Nakata, Y. Doi, Y. Hayashima, Physica D-Nonlinear Phenomena 2004, 194, 151.

15. Nakata, S.,Y. Arima, Colloids and Surfaces a-Physicochemical and Engineering Aspects 2008, 324, 222.

16. Nakata, S., Y. Doi, Y. Hayashima, Journal of Physical Chemistry B 2002, 106, 11681.

17. Nakata, S., M. Hata, Y.S. Ikura, E. Heisler, A. Awazu, H. Kitahata, H. Nishimori, Journal of Physical Chemistry C 2013, 117, 24490.

18. Nakata, S., N. Kawagishi, M. Murakami, N.J. Suematsu, M. Nakamura, Colloids and Surfaces aPhysicochemical and Engineering Aspects 2009, 349, 74.

19. Nakata, S.,K. Matsuo, Langmuir 2005, 21, 982.

20. Nakata, S., R. Tenno, Y.S. Ikura, Chemical Physics Letters 2011, 514159.

21. Restrepo-Perez, L., L. Soler, C.S. Martinez-Cisneros, S. Sanchez, O.G. Schmidt, Lab Chip 2014, 14, 1515.

22. Soh, S., K.J. Bishop, B.A. Grzybowski, J Phys Chem B 2008, 112, 10848.

23. Soler, L., V. Magdanz, V.M. Fomin, S. Sanchez, O.G. Schmidt, ACS Nano 2013, 7, 9611.

24. Wang, W., W.T. Duan, S. Ahmed, T.E. Mallouk, A. Sen, Nano Today 2013, 8, 531.

25. Wu, Y., Z. Wu, X. Lin, Q. He, J. Li, ACS Nano 2012, 6, 10910.

26. Guix, M., J. Orozco, M. Garcia, W. Gao, S. Sattayasamitsathit, A. Merkoci, A. Escarpa, J. Wang, ACS Nano 2012, 6, 4445.

27. Ikezoe, Y., G. Washino, T. Uemura, S. Kitagawa, H. Matsui, Nat Mater 2012, 11,

28. Pimienta, V.,C. Antoine, Current Opinion in Colloid \& Interface Science 2014, 19, 290.

29. Thomson, J., The London, Edinburgh, and Dublin Philosophical Magazine of Journal of Science 1855, $X$, 330.

30. Harris, C.,M. Stephens. A combined corner and edge detector. in In Alvey vision conference. 1988. Manchester, UK.

31. Zhang, Z., IEEE Trans. Pattern Analysis and Machine Intelligence 2000, 22, 1330.

32. Canny, J., IEEE Trans. Pattern Analysis and Machine Intelligence 1986, 8, 679.

33. Ioannou, D., W. Huda, A.F. Laine, Image and Vision Computing 1999, 17, 15.

34. Nakata, S., Y. Iguchi, S. Ose, M. Kuboyama, T. Ishii, K. Yoshikawa, Langmuir 1997, 13, 4454.

35. Zhao, G., T.H. Seah, M. Pumera, Chemistry 2011, 17, 12020.

36. Cassie, A.B.D.,S. Baxter, Transactions of the Faraday Society 1944, 40, 546.

37. Arneodo, C., A. Baszkin, J.P. Benoit, R. Fellous, C. Thies, Colloids and Surfaces 1988, 34, 159. 
38. Parry, E.J., The Chemistry of Essential Oils and Artificial Perfumes. 4th ed. 1922, London: Scott, Greenwood and Son.

39. Fowkes, F.M., ed. Hydrophobic surfaces. 1969, Academic Press: New York, USA.

40. Nalwa, H.S., ed. Handbook of surfaces and interfaces of materials: surface and interface phenomena. 2001, Academic Press: USA.

41. Suematsu, N.J., T. Sasaki, S. Nakata, H. Kitahata, Langmuir 2014, 30, 8101.

42. Nakata, S.,J. Kirisaka, J Phys Chem B 2006, 110, 1856.

43. Burton, L.J., N. Cheng, C. Vega, J. Andres, J.W. Bush, Bioinspir Biomim 2013, 8, 044003.

44. Nakata, S., Y. Doi, H. Kitahata, The Journal of Physical Chemistry B 2005, 109, 1798. 

Supporting Information
Click here to download S

Click here to download Supporting Information: Bio-mimetic locomotion on water of a porous natural polymeric composite Supporting Info

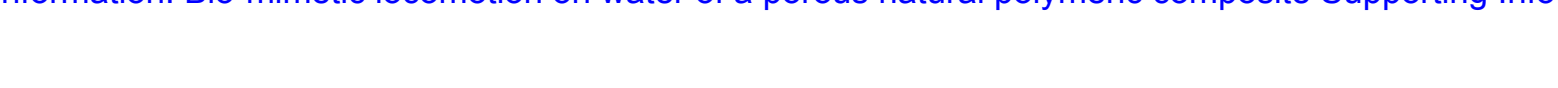

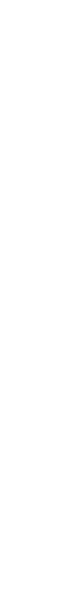

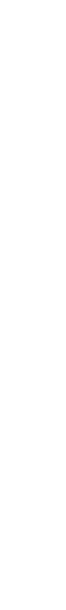

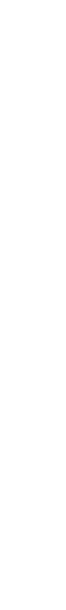
(1) (1) (1) (1) (1) (1) (1) 